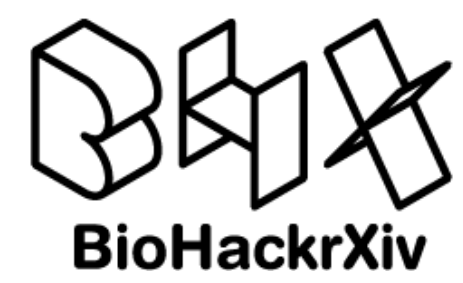

\title{
Disease and pathway maps for Rare Diseases
}

\author{
BioHackathon series: \\ BioHackathon EUROPE \\ Paris, France, 2019
}

Submitted: 20 May 2020

\section{License}

Authors retain copyright and release the work under a Creative Commons Attribution 4.0 International License (CC-BY).

Published by BioHackrXiv.org
Piotr Gawron ${ }^{1}$, David Hoksza ${ }^{1}$, Janet Piñero ${ }^{2}$, Maria Pena Chilet $^{3}$, Marina Esteban ${ }^{3}$, Jose Luis Fernandez ${ }^{3}$, Vincenza Colonna ${ }^{4}$, Joaquin Dopazo $^{3}$, Laura I. Furlong ${ }^{2}$, and Marek Ostaszewski ${ }^{1}$

1 LCSB, University of Luxembourg, Luxembourg 2 Research Programme on Biomedical Informatics (GRIB), Hospital del Mar Medical Research Institute (IMIM), Department of Experimental and Health Sciences, Pompeu Fabra University (UPF), Barcelona, Spain 3 Fundacion Progreso y Salud, Sevilla, Spain 4 Institute of Genetics and Biophysics, National Research Council of Italy

\section{Introduction}

Investigation of causal mechanisms behind Rare Diseases (RDs) is challenging, as these disorders are not prevalent enough to be represented in major bioinformatics resources or pathway databases. At the same time, a number of disease-focused resources are developed which could offer insights into specific rare diseases, if searched systematically. During BioHackathon'19 we built on an existing, rich, open source/open access infrastructure focused on disease-related mechanisms. We focused on repositories with well-defined APIs to facilitate their integration.

We streamlined different tools and platforms to enable pan-resource searches for specific questions of the field of RD research. We relied on standardized definitions and encoding of disease phenotypes, and supported the search process with high-throughput data, whenever applicable. Using these searches, we retrieved genes and variants relevant for the disease mechanisms. Using these relevant genes and variants, we identified enriched, publicly available pathway databases and disease maps, together with text mining results, to combine them into a custom disease map, generated on-the-fly. Using our workflow, a researcher is able to define an RD of choice, or encode its phenotype, to generate a relevant disease map prototype for further refinement.

\section{Approach and resources}

Our workflow can be subdivided into three, conceptually separate steps:

1. Getting data about the disease context for a given RD

2. Creating a network of known mechanisms from a set of selected repositories

3. Producing an online, interactive map prototype

All code is available in the open gitlab repository:

https://git-r3lab.uni.lu/david.hoksza/bh19-rare-diseases

\section{Disease context}

In order to introduce disease context, we focused on two major resources: OrphaNet (orpha.net) and Human Phenotype Ontology (HPO) (Köhler et al., 2017). Unique identifiers of OrphaNet allow to identify an RD, and if this disease is still not classified, it is possible to identify a proximal OrphaNet id by similarity of HPO terms.

For given OrphaNet identifiers, we obtained the list of relevant genes and variants by combining: i) gene-disease mapping of OrphaNet, ii) gene-disease and variant-disease mapping from DisGeNET (Piñero et al., 2020) (disgenet.org), iii) variant-disease mapping of OpenTargets 
platform (Carvalho-Silva et al., 2019) (opentargets.org) and iv) variant-disease mapping of ClinVar (www.ncbi.nlm.nih.gov/clinvar/). Importantly, disease-associated variants were filtered for rarity using population allele frequencies obtained from Ensembl Variant Effect Predictor (VEP) (www.ensembl.org/info/docs/tools/vep/).

In parallel, for the same OrphaNet identifiers, we searched ArrayExpress (Athar et al., 2019) (www.ebi.ac.uk/arrayexpress/) and Gene Expression Omnibus (Clough \& Barrett, 2016) (www.ncbi.nlm.nih.gov/geo/) to retrieve a list of Differentially Expressed Genes (DEGs), to extend the the set of disease-associated genes.

\section{Network of mechanisms}

The disease-relevant list of genes and variants was then used to construct a network of mechanisms using three different resources: disease maps, pathways and text mining.

Disease maps offer standardized and diagrammatic description of disease mechanisms (Mazein et al., 2018), and with the help of Gene Set Enrichment Analysis can be queried for areas of significance for a given gene list (Hoksza et al., 2019b). These areas can be exported together with their layout information, thanks to the capabilities of Systems Biology Markup Language (SBML) to support layout and render information, and functionalities of the MINERVA Platform (Hoksza et al., 2019a).

Another set of resources that support building the network of mechanisms are pathway databases. They also offer diagrammatic description of mechanisms in molecular biology, but less relevant to a particular disease. Nevertheless, they area a valuable resource and can be evaluated using enrichment. We focused on WikiPathways (Slenter et al., 2018) (wikipathways.org).

Finally, to fetch potentially novel interactions between the preselected genes, we used STRING (Szklarczyk et al., 2019) (string-db.org). Importantly, a parallel project in BioHackathon'19 focused on "Text-mining and semantic web technologies", and we worked to incorporate their outcomes into our workflow. As most of the text mining interactions are non-directional and may contain noise, we used contents of the OmniPath resource (Türei, Korcsmáros, \& Saez-Rodriguez, 2016) to increase reliability of text mining results, and to obtain directionality and sign of interactions.

\section{Interactive prototype}

As mentioned above, we chose SBML with render and layout to harmonize and integrate different resources, using the MINERVA Platform (Gawron et al., 2016), and in particular its conversion capabilities (Hoksza et al., 2019a). MINERVA was chosen as a platform to host the generated disease map, making it interactive and allowing to visually explore omics data.

To extend the use of the prototype disease map beyond visual exploration, we investigated Hipathia platform (Peña-Chilet et al., 2019)(hipathia.babelomics.org), allowing to interpret gene expression and mutation data into perturbations of signaling pathways. We focused on interface between Hipathia and MINERVA to enable analysis of the disease map, and visualization of the analysis results.

\section{Results at the BioHackathon'19}

\section{Step 1. Gathering disease-related genes and variants (seed genes)}

To obtain the disease-associated genes and variants, the workflow implements scripts to query DisGeNET, OpenTargets and ClinVar. 
Both DisGeNET and Open Targets scripts connect to the respective resources via their API and return a JSON file containing associated genes and their variants, including the association score provided by the respective platform. The scripts can be passed the required level of the association score and the maximum number of genes to be returned. The input to these scripts is a list of disease identifiers in the form of Orphanet identifiers. The Orphanet identifier is passed directly as query to Open Targets. The Ontology Lookup Service API was used to translate the Orphanet identifiers to the corresponding UMLS identifiers to query DisGeNET. All the parameters can be set in the workflow configuration files.

The list of genes and variants are then contrasted with ClinVar data which is provided as a preprocessed file. The script goes through the obtained genes and variants and carries out pairwise comparison of ClinVar non-pathogenic variants (and thus also genes) with the DisGeNET and Open Targets genes and variants. The output is a combined list of genes and variants pertinent to given disease, together with a report showing the difference in representation of genes and variants across the resources. Additionally, the variants can be filtered by their allele frequency in several populations available in the Ensembl database. This is done through the Ensemble API endpoint.

Finally, we scripted functions in $\mathrm{R}$ language to search microarrays datasets for a given rare disease in ArrayExpress and GEO databases. The user can select the organism of the datasets to be searched and filter the type/s of microarrays. Raw or normalized gene expression data can be downloaded, normalized and annotated using microarray annotation for each study. The user can choose between by study normalization or all studies as one batch normalization. Then, according to SD and QC analyses, samples are selected for the downstream processing, and, based on clinical metadata of the dataset, clinical variable is selected to assign samples to case/control groups. Finally, the script performs a meta-analysis using metaMA data and retrieves a list of differentially expressed genes (DEGs) for further analyses.

Technical issues to be considered when calculating DEGs from GEO and ArrayExpress:

- Missing clinical/phenotype data from datasets prohibits group definition for DEGs calculation.

- The variability of the platforms and technologies for MicroArrays studies is challenging for systematic translation of probes to gene ids and for automatic data retrieval.

- Data normalization and QC cannot be fully automated.

- In order to perform meta-analysis, data must be homogeneously normalized, that implies that only raw datasets should be retrieved.

- The definition of case/control groups must be made manually, and often needs from clinical and disease knowledge.

\section{Step 2. Assembly and extension of network of mechanisms}

We expanded the number of disease-associated genes and variants (seed genes), assembled in previous steps, using resources as described above. The script receives as an input a list of seed genes (from OrphaNet, DisGeNET, OpenTargets and ClinVar) and expands the list of seed genes using information from different resources.

\section{Disease maps}

Disease maps hosted on MINERVA can be queried via API calls, and their content can be retrieved, including layout and network structure. We used Parkinson's disease map (pdmap.uni.lu) as an example in our workflow. Nevertheless, source disease maps, if they are open access, can be set in configuration files of the workflow. For each selected disease map, the workflow performs Gene Set Enrichment Analysis for the seed genes (for GSEA in MINERVA see (Hoksza et al., 2019b)) and indicates enriched areas for later integration. 
Pathway databases

We used the enrichR package, an R-based interface to the Enrichr server (Kuleshov et al., 2016) to calculate pathway enrichment for the seed genes. As enrichR allows for querying multiple packages at once, a configuration file (see below) allows to indicate, which pathway databases can be used. Currently, our workflow handles only the contents of Wikipathways.

Text mining and interaction databases

\section{STRING}

STRING integrates primary and predicted interactions, includes annotated pathway knowledge, text-mining results and data obtained by ontology. The query to STRING retrieves the first $\mathrm{n}$ neighbors of the seed genes. The number of neighbors can be adjusted by modifying the configuration of the workflow (see below). Moreover, the score for the associations among the seed proteins and the extended list of proteins can be set by modifying the parameter score in the configuration file. By default, the score equals zero.

\section{OmniPath}

The query to OmniPath retrieves all associations in the database for the seed genes. The information from OmniPath includes directionality (values $=1$ means direction, value $=0$ means no direction). The column consensus_directionality reflects the fact that some evidences may indicated the directionality to be from protein $A$ to protein $B$ for a given pair, and vice versa. We also extracted from OmniPath the references supporting the relationship between the pairs of proteins. The information from STRING is enriched by the input from OmniPath to provide directionality to those pairs of proteins reported by both resources.

\section{Step 3. Integration of map pieces into a prototype}

We implemented functionality to integrate all the pieces assembled in Step 2, namely disease map segments, pathways and text mining data.

Disease map segments, identified via GSEA for the seed genes, were retrieved from respective disease maps. Because this content is hosted by MINERVA, these pieces have their layout and rendering information fully preserved.

MINERVA Platform ( $v 15$, currently in beta) is capable of importing and exporting GPML format, used by Wikipathways. This allows for programmatic handling of pathways enriched in Step 2. Because of the translation between two different layout and rendering descriptions, some details, e.g. custom images or interactions without reactant/product, are lost.

Text mining information has inherently no layout nor rendering information. For these elements, a circular layout was introduced.

The newly implemented functionality integrated the pieces from Step $\mathbf{2}$ in a mesh-like layout. See image below for an example. In the image, Wikipathways content is in the upper part of the map, text mining results in the middle, and the content from disease maps at the bottom. 

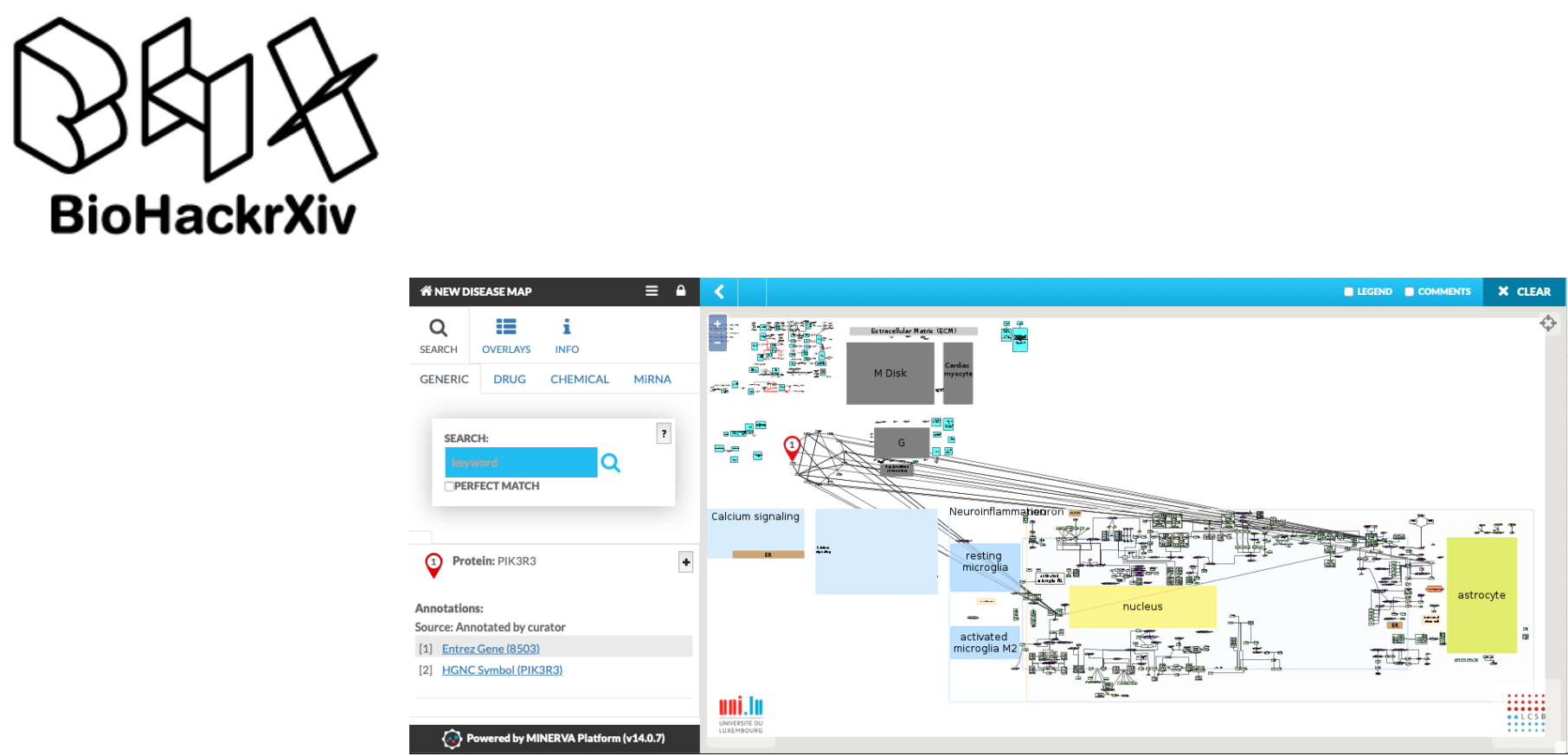

Figure 1: An example map generated for the Brugada syndrome based on its Orphanet identifier.

Moreover, we worked on an interface from MINERVA to a full pathway-based analysis and visualization of disease maps in HiPhathia web tool. The task is divided in back-end and front-end related work. The back-end was developed under the Java EE platform, using Java 11 and the Spring Framework plus Spring Batch for managing the job queue, and a Mongo database for data persistence. The front-end was prototyped using TypeScript and ReactJS.

\section{Back-end}

First, a service with a RESTful interface takes an identifier from MINERVA Platform network and a RD expression dataset as inputs, decompresses and stores the files in a temporal folder and queues the files to the second service, returning a job identifier. Second, a Mongo and Spring Batch based service downloads MINERVA-hosted disease map using dedicated services, developed during the BioHackathon. The map is preprocessed for its use in HiPhathia web tool. Then, HiPathia algorithm for pathway-based analysis is executed as an R script. Finally, a HTTP server is prepared for the visualization of the results from HiPathia pathway-based analysis, with an URL based on the job identifier.

Front-end We developed a web component for its inclusion in Hipathia Web (and possibly in MINERVA) allowing the user to upload experimental data and select a MINERVA disease map, execute the corresponding Hipathia analysis, and view the results.

The existing implementation of Hipathia serves the results over HTTP directly from R. Currently, this is hardwired in code, which lacks sophistication to specify multiple URIs for multiple analyses, and is challenging for our Java-based architecture. This was circumvented with temporary workaround by copying the results to an existing Apache HTTP server folder. A more satisfying solution requires evolving the related Hipathia function.

\section{Step 4. Streamlining of the previous steps and configuration of the workflow}

The workflow is implemented as a shell pipeline which can be configured by a parameters file where the user can set all the parameters mentioned above including the list of disease identifiers. The output of the pipeline is a ZIP file containing the disease map with genetic and variants overlays and can be imported into MINERVA. The pipeline implements the above described steps in the following order:

1. Obtain gene-disease and variant-disease mapping from DisGeNET.

2. Obtain gene-disease and variant-disease mapping from OpenTargets.

3. Obtain possibly pathogenic ClinVar variants and genes pertinent to given disease.

4. Compile list of of genes associated with disease from all the input sources.

5. Extend the list of genes by going to other resources such as OmniPath or text mining. 


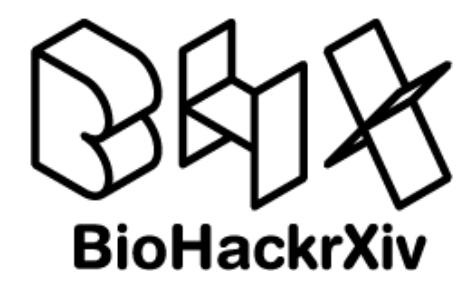

6. Compile list of of variants associated with disease from all the input sources.

7. Filter out variants with high allele frequency using Ensemble's VEP service.

8. Obtain variant information (position, protein-level mapping) and store it for MINERVA genetic variant overlay.

9. From resources such as existing disease maps or WikiPathways obtain enriched pathways with respect to the disease-associated genes obtained from previous step.

10. Compile the obtained pathways into a single disease map.

11. Bundle the disease map with genetic and variant overlays into a single archive to be then uploaded to MINERVA.

\section{Summary and outlook}

The workflow integrating disease and pathway maps according to a given RD identifier may be helpful for RD researchers in setting up their respective knowledge repositories, and mapping their datasets. There are a number of improvements we foresee for this work, including using SPARQL query of Wikipathways for more accurate enrichment, deeper integration with text mining service developed during the BioHackathon'19, or improvement of the Hipathia interface.

The workflow can be expanded on each step, adding i) new gene candidate resources, ii) new pathway databases or iii) introducing layout for text mining results and broaden the downstream map analysis. We will continue our work to explore these directions.

\section{Acknowledgements}

This work was done during the The BioHackathon Europe, organised and funded by the ELIXIR Hub in November 2019 in Paris. We thank the organizers for an opportunity to participate in such a productive and collaborative event, and for accomodation.

We thank Steve Laurie, Centro Nacional de Análisis Genómico (CNAG-CRG), Barcelona, Spain, for support in the domains of rare disesase phenotyping and genomics.

We thank colleagues from the "Text-mining and semantic web technologies" project for developing dedicated API calls and advising on the use of text mining resources.

\section{References}

Athar, A., Füllgrabe, A., George, N., Iqbal, H., Huerta, L., Ali, A., Snow, C., et al. (2019). ArrayExpress update - from bulk to single-cell expression data. Nucleic Acids Research, 47(D1), D711-D715. doi:10.1093/nar/gky964

Carvalho-Silva, D., Pierleoni, A., Pignatelli, M., Ong, C., Fumis, L., Karamanis, N., Carmona, M., et al. (2019). Open targets platform: New developments and updates two years on. Nucleic Acids Research, 47(D1), D1056-D1065. doi:10.1093/nar/gky1133

Clough, E., \& Barrett, T. (2016). The gene expression omnibus database. Methods in Molecular Biology (Clifton, N.J.), 1418, 93-110. doi:10.1007/978-1-4939-3578-9_5

Gawron, P., Ostaszewski, M., Satagopam, V., Gebel, S., Mazein, A., Kuzma, M., Zorzan, S., et al. (2016). MINERVA-a platform for visualization and curation of molecular interaction networks. NPJ systems biology and applications, 2, 16020. doi:10.1038/npjsba.2016.20

Hoksza, D., Gawron, P., Ostaszewski, M., Hausenauer, J., \& Schneider, R. (2019a). Closing the gap between formats for storing layout information in systems biology. Briefings in Bioinformatics. doi:10.1093/bib/bbz067 
Hoksza, D., Gawron, P., Ostaszewski, M., Smula, E., \& Schneider, R. (2019b). MINERVA $\mathrm{API}$ and plugins: Opening molecular network analysis and visualization to the community. Bioinformatics (Oxford, England), 35(21), 4496-4498. doi:10.1093/bioinformatics/btz286

Köhler, S., Vasilevsky, N. A., Engelstad, M., Foster, E., McMurry, J., Aymé, S., Baynam, G., et al. (2017). The human phenotype ontology in 2017. Nucleic Acids Research, 45(D1), D865-D876. doi:10.1093/nar/gkw1039

Kuleshov, M. V., Jones, M. R., Rouillard, A. D., Fernandez, N. F., Duan, Q., Wang, Z., Koplev, S., et al. (2016). Enrichr: A comprehensive gene set enrichment analysis web server 2016 update. Nucleic Acids Research, 44(W1), W90-97. doi:10.1093/nar/gkw377

Mazein, A., Ostaszewski, M., Kuperstein, I., Watterson, S., Le Novère, N., Lefaudeux, D., De Meulder, B., et al. (2018). Systems medicine disease maps: Community-driven comprehensive representation of disease mechanisms. NPJ systems biology and applications, 4, 21. doi:10.1038/s41540-018-0059-y

Peña-Chilet, M., Esteban-Medina, M., Falco, M. M., Rian, K., Hidalgo, M. R., Loucera, C., \& Dopazo, J. (2019). Using mechanistic models for the clinical interpretation of complex genomic variation. Scientific Reports, 9(1), 18937. doi:10.1038/s41598-019-55454-7

Piñero, J., Ramírez-Anguita, J. M., Saüch-Pitarch, J., Ronzano, F., Centeno, E., Sanz, F., \& Furlong, L. I. (2020). The DisGeNET knowledge platform for disease genomics: 2019 update. Nucleic Acids Research, 48(D1), D845-D855. doi:10.1093/nar/gkz1021

Slenter, D. N., Kutmon, M., Hanspers, K., Riutta, A., Windsor, J., Nunes, N., Mélius, J., et al. (2018). WikiPathways: A multifaceted pathway database bridging metabolomics to other omics research. Nucleic Acids Research, 46(D1), D661-D667. doi:10.1093/nar/gkx1064

Szklarczyk, D., Gable, A. L., Lyon, D., Junge, A., Wyder, S., Huerta-Cepas, J., Simonovic, M., et al. (2019). STRING v11: Protein-protein association networks with increased coverage, supporting functional discovery in genome-wide experimental datasets. Nucleic Acids Research, 47(D1), D607-D613. doi:10.1093/nar/gky1131

Türei, D., Korcsmáros, T., \& Saez-Rodriguez, J. (2016). OmniPath: Guidelines and gateway for literature-curated signaling pathway resources. Nature Methods, 13(12), 966-967. doi:10. $1038 /$ nmeth.4077 